\title{
Characterization of malignant brain tumor using elastic light scattering spectroscopy
}

\author{
Jianmin Gong ${ }^{\mathrm{a}}$, Ji Yi ${ }^{\mathrm{a}}$, Vladimir M. Turzhitsky ${ }^{\mathrm{a}}$, Kenji Muro ${ }^{\mathrm{b}}$ and $\mathrm{Xu} \mathrm{Li}^{\mathrm{a}, \mathrm{c}, *}$ \\ ${ }^{a}$ Department of Biomedical Engineering, Northwestern University, Evanston, IL 60208, USA \\ ${ }^{\mathrm{b}}$ Department of Neurological Surgery, Northwestern University, Chicago, IL 60611, USA \\ ${ }^{\mathrm{c}}$ Department of Electrical Engineering and Computer Science, Evanston, IL 60208, USA
}

\begin{abstract}
We report a pilot study designed to test elastic light-scattering (ELS) spectroscopy for characterizing normal, tumor, and tumor-infiltrated brain tissues. ELS spectra were measured from 393 sites on 36 ex vivo tissue specimen obtained from 29 patients. We employed and compared the performances of three methods of spectral classification for tissue characterization, including spectral slope analysis, principle component analysis (PCA), and artificial neural network (ANN) classification. The ANN classifier yielded the best correlation between spectral pattern and histopathological diagnosis, with a typical sensitivity of $80 \%$ and specificity of $93 \%$ for differentiating tumor from normal brain tissues. We also demonstrate that all three classification methods discriminate between tumor and normal tissue and have the potential to identify and quantitatively characterize tumorinfiltrated brain tissues.
\end{abstract}

Keywords: Brain cancer, glioma, elastic light scattering spectroscopy, spectral slope, principle component analysis, artificial neural network

\section{Introduction}

Malignant brain tumors continue to trouble the neuro-oncology community with their poor prognosis despite aggressive surgical and adjuvant therapy [1]. Initial surgical intervention remains the standard of care for these patients, which accomplishes several goals: to establish histopathological diagnosis, to relieve mass effect from adjacent brain, and to cytoreduce, or decrease the tumor burden, in preparation for postsurgical adjuvant therapy. One of the variables shown to result in longer patient survival and improved quality of life after the diagnosis of malignant brain tumor is maximal tumor resection at the time of surgery $[2,3]$. Thus, neurosurgeons have sought tools that will complement their own intraoperative clinical impression to help achieve maximal resection while maintaining patient safety and neurological function.

Many resources are currently available to the neurosurgeon to optimize operative resection; however, each

\footnotetext{
*Corresponding author: Xu Li, E-mail: xuli@ northwestern.edu.
}

strategy has limitations with regard to sensitivity, resolution, cost, and additional surgical time. For example, the intraoperative ultrasonography machine is a fairly inexpensive tool and offers the surgical team real-time anatomic analysis capabilities; however, it is hampered by the lack of sensitivity and resolution, especially adjacent to dense bony structures [4-7]. With the advent of the computed tomography (CT), many investigators studied its utility in the operating room. However, the intraoperative CT units were costly to install, had limited soft tissue discrimination, and exposed the surgical team to $\mathrm{x}$-radiation [8-11]. The magnetic resonance imaging (MRI) technology offers superior soft tissue resolution than ultrasonography or CT, and real-time surgical navigation tools were developed that allowed surgeons to verify the position of a surgical instrument in relation to structures seen on the imaging study [12]. Nevertheless, due to the static nature of the image, acquired prior to commencing surgery, the navigation system lost reliability with the onset of brain "shift" during surgery [13], at times resulting in shift of tumor margins by more than a centimeter in imaging space 
compared to their actual locations [5]. In 1999, utility of the first-generation intraoperative MRI unit was reported. This technology brought the most sophisticated imaging modality to the operating room; however, it has not gained widespread use, owing to its initial profound cost, time required to acquire the images, and somewhat limited resolution due to smaller magnetic coils compared to conventional diagnostic MRI machines [14-17]. Lastly, the traditional method of consulting the pathologist to analyze surgical specimen remains an important but labor-intensive option that also requires the repeated removal of tissue, lengthy waiting periods, variable inter-observer reliability, and cost. Therefore, the innovation of a novel technique that reliably discriminates tumor from normal tissues while allowing real-time analysis of the tissue in the operating room would be a valuable contribution to patient care.

At the same time, for one of the most challenging categories of brain tumors, the malignant glioma, the therapeutic failure is also to a large extent due to the diffuse growth pattern of this cancer, which infiltrates extensively into the surrounding brain tissue. As significant research effort is underway to understand the pattern and molecular mechanism of glioma cell migration, in vivo identification and imaging of the infiltrating front of the tumors may have important prognostic and therapeutic implications. Currently, however, radiographic visualization of the tumor-infiltrated brain region is extremely difficult, often resulting in significant underestimation of the extent of glioma growth [18,19]. The inaccurate estimation of the infiltration makes it difficult to make patient-specific optimized decisions during both surgical intervention and adjuvant therapy. Postsurgically, there are non-invasive imaging modalities being studied to tailor adjuvant therapy that may also help predict the pattern of recurrent glioma [20]. Thus, if regions at highest risk for recurrent tumor can be identified intraoperatively, it would again contribute significantly to surgeons' decision-making process and to the patients' care.

It has been extensively demonstrated that intrinsic optical properties of biological tissue can be utilized to conduct in vivo tissue characterization for several organs [21-31]. Optical techniques that have been developed in the past decades to characterize tumor and normal brain tissue include optical coherence tomography [32-34], optical imaging enhanced with fluorescent dyes and other contrast agents [35-39], autofluorescence spectroscopy [40-42], and a combination of autofluorescence and diffuse reflectance spectroscopy [43-46]. In particular, a hand-held optical spectroscopic probe was developed to measure autofluorescence and diffuse reflectance spectra to intraoperatively characterize solid tumor and infiltrating tumor margins [46]. These initial measurements strongly indicate the feasibility of using intrinsic optical properties as contrast mechanism for characterizing brain tissues.

In this paper, we explore the use of elastic lightscattering spectroscopy (ELS) to characterize normal, tumor, and tumor-infiltrated brain. Compared to diffuse reflectance spectroscopy, the ELS technique has the distinctive feature of measuring single-scattering photons, instead of measuring photons that have been scattered multiple times. It has been observed that single-scattering photons, which are the main contribution of ELS spectra, are affected by tissue architectures in the micro-nano scales, and therefore offers the potential to provide quantitative information about biological structures without the need for tissue biopsy, fixation and staining, or other processing. We have previously theoretically demonstrated how backscattering spectra are sensitive to nanoscale perturbations at scales from tens of nanometers to microns $[47,48]$, thus encompassing a spectrum of structures ranging from macromolecular complexes to whole cells. Promising early-stage clinical research on colon carcinogenesis has also demonstrated the feasibility of detecting carcinogenesis far earlier than conventional strategies using ELS signatures $[29,30,49,50]$, which further indicates the sensitivity afforded by the ELS technique. Interestingly, ELS appears to provide quantitative characterization of the progression of carcinogenesis [30, 50] and therefore offers the potential to quantify the infiltrating region of diffuse glioma, where tumor cells tend to invade individually or in small groups into the surrounding tissue, away from the main tumor mass.

In the study presented in this paper, we measured ELS spectra of ex vivo tissue specimen for a variety of brain tissues and correlated the spectral features with different tissue types. We employed and compared the performance of three methods of spectral classification for tissue characterization, including spectral slope analysis, principle component analysis (PCA), and artificial neural network (ANN) classification. As demonstrated in Section 3, the ANN classifier yielded the best correlation between spectral pattern and histopathological diagnosis, with a typical sensitivity of $80 \%$ and specificity of $93 \%$ for differentiating tumor from normal brain tissue. We also demonstrate that all three classification methods have the potential to identify and characterize tumor-infiltrated brain tissue. 


\section{Materials and methods}

Ex vivo brain tissue specimen were collected by surgeons in the Department of Neurological Surgery at Northwestern University Feinberg School of Medicine. Brain tissues were excised during elective cranial surgery and transferred to the Biomedical Engineering Department for ELS measurement. The protocol of this study was approved by the Northwestern University Institutional Review Board. Data analysis and classification based on ELS spectra were compared with histopathological diagnosis.

\subsection{Specimen preparation}

Tumor specimen (brain tumor and tumor-infiltrated brain) and normal brain matter were obtained from patients undergoing surgical treatment for brain tumor or epilepsy. The specimen were collected after informed consent was obtained for this study and for the Northwestern University Feinberg School of Medicine PathCORE tumor banking program. We collected 25 brain tumors, 8 normal brain and 3 tumor-infiltrated brain specimen from 29 patients, as shown in Table 1. All specimen were kept in $0.9 \%$ saline immediately after excision and stored at $4{ }^{\circ} \mathrm{C}$. Optical measurement was conducted in the Biomedical Engineering Department within $48 \mathrm{~h}$ after resection. After measurement, the specimen were fixed in $10 \%$ formalin solution.

\subsection{ELS measurements}

The system we used to conduct the ELS measurements is similar as described in [49]. Briefly, as Fig. 1 shows, a collimated light source (LS) was polarized by a linear polarizer (P1). The sample was illuminated over an approximate area of $2 \mathrm{~mm}^{2}$. The light scattered by the sample is collected by a lens (L1). A second polarizer (P2) is used to select the polarization state of the scattered light so that the co-polarized component $\left(I_{||}\right)$and the cross-polarized component $\left(I_{\perp}\right)$ of the backscattered light could be recorded separately. The entrance slit of the spectrograph SP2150 (Princeton Instrument, Inc) is placed on the focal plane of the lens, which focuses light from different scattering angles to the entrance slit. The system covered visible light ranging from 400-700nm in wavelength. A CoolSnap CCD camera (Princeton Instrument, Inc) is used to collect a 2D map of scattering intensity with the $\mathrm{x}$ axis as wavelength $(\lambda)$ and $y$ axis as scattering angle. The exposure time was set as $5 \mathrm{~s}$. A single spectrum was obtained

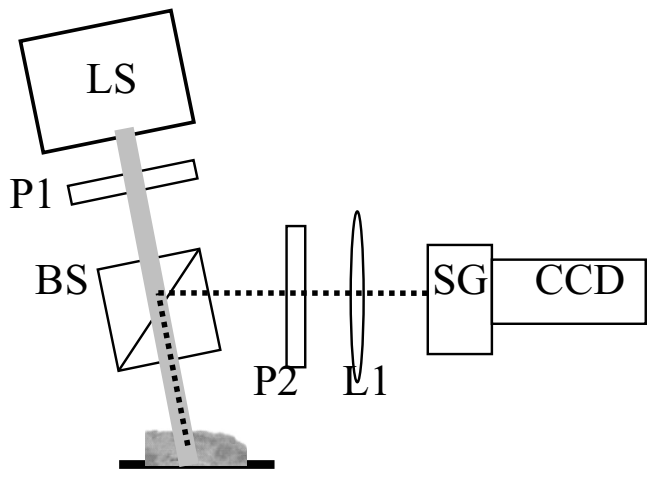

Fig. 1. ELS system configuration. Collimated white light coming from light source (LS) module was linearly polarized by polarizer (P1) and then illuminated brain specimen. Elastic light scattered was redirected by beam splitter (BS) and gated by another polarizer (P2). The distance between lens (L1) and spectrograph was a focal length then L1 focused light scattered from different zenithal angle to entrance slit of SG, which further disperse light into spectrum. Therefore, $\mathrm{CCD}$ collected 2D frame with respect to scattering zenithal angle and wavelength.

for each exposure by averaging the scattering map for an angular range of $4^{\circ}$ centered at the backscattering direction. Depending on the size of the specimen, we made 3 to 20 backscattering measurements at different spots in order to make as much coverage as possible, recording both co-polarized and cross-polarized spectra for each. Background spectra while no sample was placed, and white standard backscattering spectra were recorded and used to minimize the effect of stray light and limited spectral bandwidth of the system. Background spectra was recoded without sample and subtracted from raw spectra to exclude the effect of stray light; and then white standard backscattering spectra were recorded to characterize the spectral profile of the light source.

\subsection{Preprocessing of light scattering spectra}

After the background extraction and white standard normalization, we obtained the co-polarized and crosspolarized backscattering spectra $\left(I_{\|}(\lambda)\right.$ and $\left.I_{\perp}(\lambda)\right)$ from the specimen. Then we took several steps to preprocess the spectra so that they are suitable for further analysis. First, we applied polarization gating to selectively enhance contribution from single-scattering photons. This can be simply achieved by subtracting the cross-polarized spectrum $I_{\perp}(\lambda)$ from the corresponding co-polarized spectrum $I_{||}(\lambda)$ based on the fact that multiple scattering depolarizes light $[49,51,52]$.

Next, we removed the effect of hemoglobin absorption features from the spectra. There is usually 
Table 1

Types and quantities of brain tissue samples

\begin{tabular}{lcccc}
\hline Disease & $\begin{array}{c}\text { Number } \\
\text { of patients }\end{array}$ & $\begin{array}{c}\text { Number of } \\
\text { tumor samples }\end{array}$ & $\begin{array}{c}\text { Number of } \\
\text { normal samples }\end{array}$ & $\begin{array}{c}\text { Number of } \\
\text { infiltrating samples }\end{array}$ \\
\hline Tumor - glioma & 17 & 17 & 2 & 3 \\
Tumor - metastasis & 6 & 6 & 2 & 0 \\
Tumor - nonglial & 2 & 2 & 0 & 0 \\
Seizure & 4 & 0 & 4 & 0 \\
Total & 29 & 25 & 8 & 3 \\
\hline
\end{tabular}

blood remaining on the tissues to be tested, and the hemoglobin component introduces absorption spectral features, which may affect the spectral features used for diagnostics and thus should be removed before further analysis. The two types of hemoglobin concerned in our tests - namely the oxygenated hemoglobin $\left(\mathrm{HbO}_{2}\right)$ and deoxygenated hemoglobin $(\mathrm{Hb})$ - generate distinct absorption features throughout the spectral range of $500-600 \mathrm{~nm}$ in the backscattering spectra. As it has been previously observed that attenuation due to absorption of both hemoglobin components have an inverse exponential relationship to their concentration [53], the back-scattered spectrum from a small portion of the bloody sample can be approximated as [53]:

$$
\mathbf{i}(\lambda)=(a \lambda+b) \exp [-o \mathbf{O}(\lambda)-d \mathbf{D}(\lambda)]
$$

where $o, \mathbf{O}, d$ and $\mathbf{D}$ are the concentration and spectral absorption coefficient of $\mathrm{HbO}_{2}$ and $\mathrm{Hb}$, respectively, and $a, b$ are coefficients determined by the spectral characteristics of the source, detector and sample scattering properties. Since the backscattered light we detected originates from a range of depths and lateral positions inside of the specimen, we model the corresponding spectrum as a summed contribution of multiple $\mathbf{i}(\lambda)$ 's:

$$
\begin{aligned}
\mathbf{I}(\lambda)= & \sum_{j}\left(a_{j} \lambda+b_{j}\right) \\
& \quad \exp \left[-o_{j} \mathbf{O}(\lambda)-d_{j} \mathbf{D}(\lambda)\right]
\end{aligned}
$$

We used 2nd-order Taylor series to approximate this $I(\lambda)$ while neglecting higher-order terms, with the practical assumption that $o \mathbf{O}<<1, d \mathbf{D}<<1$.

$$
\begin{aligned}
& \mathbf{I}(\lambda) \approx p_{00}+p_{01} \lambda+p_{10} \mathbf{O}+p_{11} \mathbf{D}+p_{12} \mathbf{O} \lambda \\
& +p_{13} \mathbf{D} \lambda+p_{20} \mathbf{O}^{2}+p_{21} \mathbf{D}^{2}+p_{22} \mathbf{O D} \\
& +p_{23} \mathbf{O}^{2} \lambda+p_{24} \mathbf{D}^{2} \lambda+p_{25} \mathbf{O D} \lambda
\end{aligned}
$$

Here the unknown coefficients $p_{x x}$ can be estimated using min-variance optimal solution from the equation by discretizing the measured spectra in wavelength range of 500-600 nm, and the hemoglobin-free spectrum is calculated by applying calculated $p_{10} \sim p_{25}$ to the whole spectral range (e.g. $460-670 \mathrm{~nm}$ for our tests):

$$
\begin{aligned}
& \mathbf{I}_{n o H b}(\lambda)=\mathbf{I}(\lambda)-p_{10} \mathbf{O}-p_{11} \mathbf{D}-p_{12} \mathbf{O} \lambda \\
& -p_{13} \mathbf{D} \lambda-p_{20} \mathbf{O}^{2}-p_{21} \mathbf{D}^{2}-p_{22} \mathbf{O D} \\
& -p_{23} \mathbf{O}^{2} \lambda-p_{24} \mathbf{D}^{2} \lambda-p_{25} \mathbf{O D} \lambda
\end{aligned}
$$

Finally, all the $\mathbf{I}_{n o H b}(\lambda)$ were normalized to unit power to eliminate the effect of the fluctuation of source power, hemoglobin absorption loss and CCD sensitivity.

\subsection{Methods of data analysis}

We employed and compared the performance of three methods of spectral classification for brain tissue characterization based on the ELS measurements, including spectral slope analysis, principle component analysis (PCA), and artificial neural network (ANN) classification.

Spectral slope of ELS spectra has been associated with intracellular particle size distribution and density of intracellular particles ranging from macromolecules to organelles $[21,30,31,50]$ and have been demonstrated to be a quantitative marker for tissue characterization. After preprocessing, we divided each ELS spectrum measured from the tissue samples to five relatively linear segments with wavelength ranges of 460$480 \mathrm{~nm}, 480-520 \mathrm{~nm}, 520-560 \mathrm{~nm}, 560-600 \mathrm{~nm}$ and $600-670 \mathrm{~nm}$. The spectral slope for each spectrum segment was calculated as the coefficient of the linear fit to the spectrum. During data analysis, we observed that spectral slope of the $600-670 \mathrm{~nm}$ segment gave the most accurate classification of tissue types, and therefore this wavelength range was empirically chosen for statistical analysis for diagnosis.

Principal components analysis (PCA) may effectively reduce multidimensional data sets to lower dimensions, and has been widely used in diagnosis when there is no definite physical model available to describe the data. The spectral principal components for all the preprocessed spectra were calculated with Matlab statistics toolbox version 7.5 (The Mathworks, Inc). Here, the entire measured spectral range $(400-700 \mathrm{~nm})$ was used and each spectrum was represented as a set of intensities (PCA variables) as input, and the projections 
Table 2

Comparison of performance for spectral slope, PCA, and ANN methods

\begin{tabular}{llll}
\hline & Spectral slope & Projection to PC1 & ANN leave-one-sample \\
\hline AUC & 0.78 & 0.72 & 0.92 \\
(Sensitivity,specificity) & $(80 \%, 63 \%)$ & $(80 \%, 49 \%)$ & $(80 \%, 93 \%)$ \\
\hline
\end{tabular}

of the preprocessed spectra to different spectral principal components were used as the scores to distinguish tumor and normal brain samples.

For classification involving complex and nonlinear models, ANN has been demonstrated to have superior performance due to its versatility and ability to be customized to specific applications and its ability to cope with nonlinear problems [21]. Here, we implemented a 3-layered feed-forward ANN to estimate the relation between the spectral features and the histopathological diagnosis. The band of 460-670 nm was divided into ten equal segments, and the mean of each segment corresponds to one nodes in the input layer of the ANN. There are, in total, 10 nodes in the input layer. The number of nodes in the middle layer was set to seven after trial tests, since the corresponding ANN is complicated enough to approximate the target input-output relation, and is not too complicated to suppress the input noise. The output layer contains a single node which represents the type of the sample and is pre-defined as 0 for normal sample and 1 for tumor sample. An input for the ANN contains a preprocessed ELS spectrum, represented by a ten-element array from the mean intensities of its ten segments. For ANN training, each input also contains a score from the known histopathological diagnosis. A back-propagation gradient descent algorithm with momentum term (implemented with Matlab 7.0 ANN toolbox) was used to train the ANN iteratively to minimize the error between the pre-defined output scores and the simulated output scores. After training, we estimated the performance of the trained ANN by comparing ANN output with histopathological diagnosis for test data set containing ELS spectra that have not been used for the ANN training.

\section{Results}

The performance of three methods of spectral classification for brain tissue characterization described in 2.4 is summarized in Figs 2-4. Figure 2(a) shows representative ELS spectra of tumor/normal/tumorinfiltrated samples obtained from a single patient. Difference of the spectral slopes can be visually noted for these three tissue types. The box plot of the spectral slopes of all the preprocessed spectra from the same pa- tient are shown in Fig. 2(b). The means of the spectral slopes for tumor and normal samples separate significantly, and the mean of the spectral slopes for tumorinfiltrated samples falls in between. The box plot of the spectral slopes of the spectra from all the samples is shown in Fig. 2(c). There is more overlapping between the slopes of tumor and normal samples, since the between-patient variations are increased for the tissue type. Similar to the single-patient analysis, the tumor-infiltrated-sample slopes fall between those of tumor and normal brain samples.

According to the statistics of the ELS spectral slopes, a slope threshold may be used to classify the sample type: a spectrum is regarded as from a tumor sample if the spectral slope of the spectrum is less than the threshold; and as from normal sample otherwise. For each slope threshold we may get a diagnostic sensitivity and specificity when comparing the classification to histopathological diagnosis. By changing the threshold, a set of sensitivity and specificity values can be calculated. The Receiver Operating Characteristic (ROC) curve is shown as Fig. 2(d) in which the values of Sensitivity are drawn v.s. (1-Specificity). The area under the curve (AUC) of the ROC curve is 0.78 , and a typical pair of sensitivity and specificity is $80 \%$ and $63 \%$.

For PCA analysis, projections to principal component (PC) 1, i.e. PC1 scores, were used as the classification marker for tissue types. Figure 3(a) shows the spectrum of the PC1 component. The PC1 projection scores from different tissue types are shown in Fig. 3(b) for same-patient analysis and Fig. 3(c) for all-patients analysis. Similar to spectral slope marker, the PC1 scores for tumor-infiltrated brain samples fall between those for tumor and normal samples. The ROC curve of the PC1 score marker is shown as Fig. 3(d). Its AUC value is 0.72 , and a typical pair of sensitivity and specificity is $80 \%$ and $49 \%$.

ANN analysis for tissue classification is shown in Fig. 4. Figure 4(a) shows the result of a leave-half test, i.e., ELS spectra from half of the tumor/normal brain sample population (randomly chosen) were used to train the ANN, and the other half were used for testing. The bold curve gives the preset scores, either 0 or 1 , and the thin curve is the output score for tests. It is obvious that when the training data set was used 

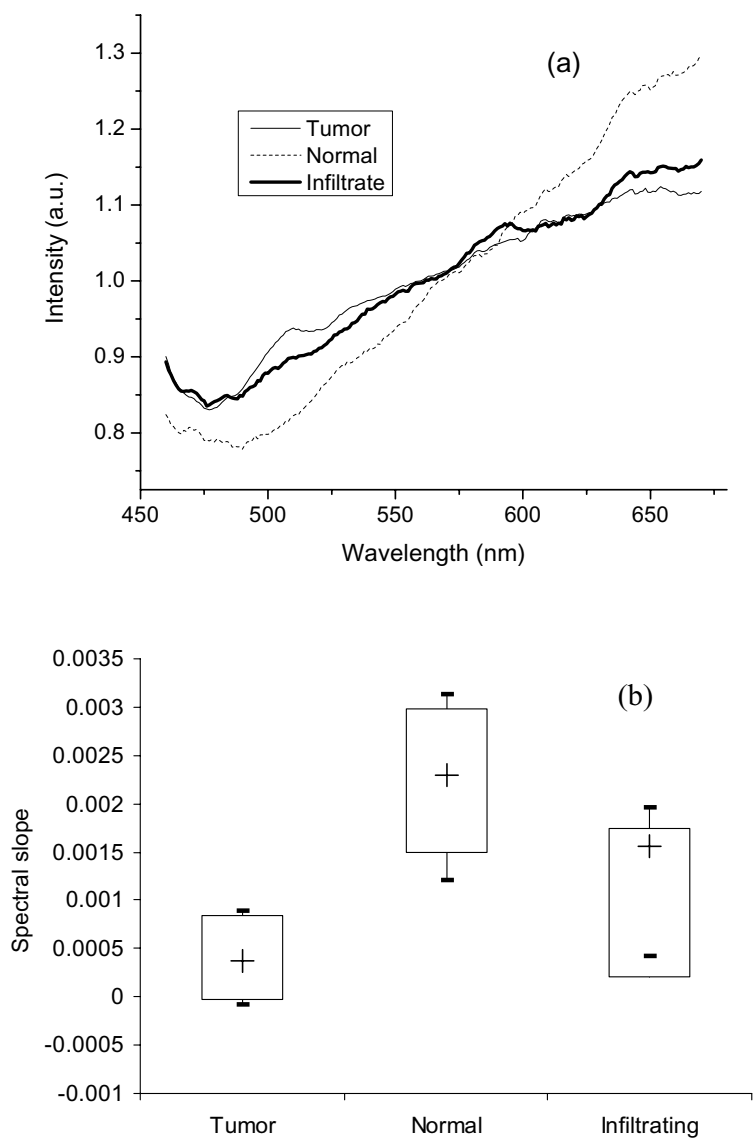

Fig. 2a, b. ELS spectra and statistical analysis of spectral slopes for three types of brain tissue. (a) Typical ELS spectra for normal brain tissue, tumor, and tumor-infiltrated brain tissue. The three types of tissue samples were obtained from a single patient. (b) Statistics of ELS spectral slopes for three tissue types from a single patient. Box: 1 and 3 quartiles; bars: mean \pm standard deviation; cross: median. (c) Statistics of ELS spectral slopes of the spectra from all the patients as listed in Table 1. (d) ROC curve of using spectral slope as a diagnostic marker

again for testing, the output scores are usually consistent with the preset scores corresponding to tissue histopathology. This proves the self-consistency and stability of the trained ANN. When testing for the other half sample population which were not used as training data, the output scores are also usually close to the 0/1 scores corresponding to the tissue histopathology, demonstrating that these output scores are capable of predicting the samples histopathology. For the purpose of diagnosis, a threshold may be introduced where an ELS spectrum is regarded as from a tumor sample if the simulated score is larger than the threshold; and otherwise as from a normal sample.

We adopted a leave-one-sample test scheme to evaluate the performance of the ANN classification. Here,
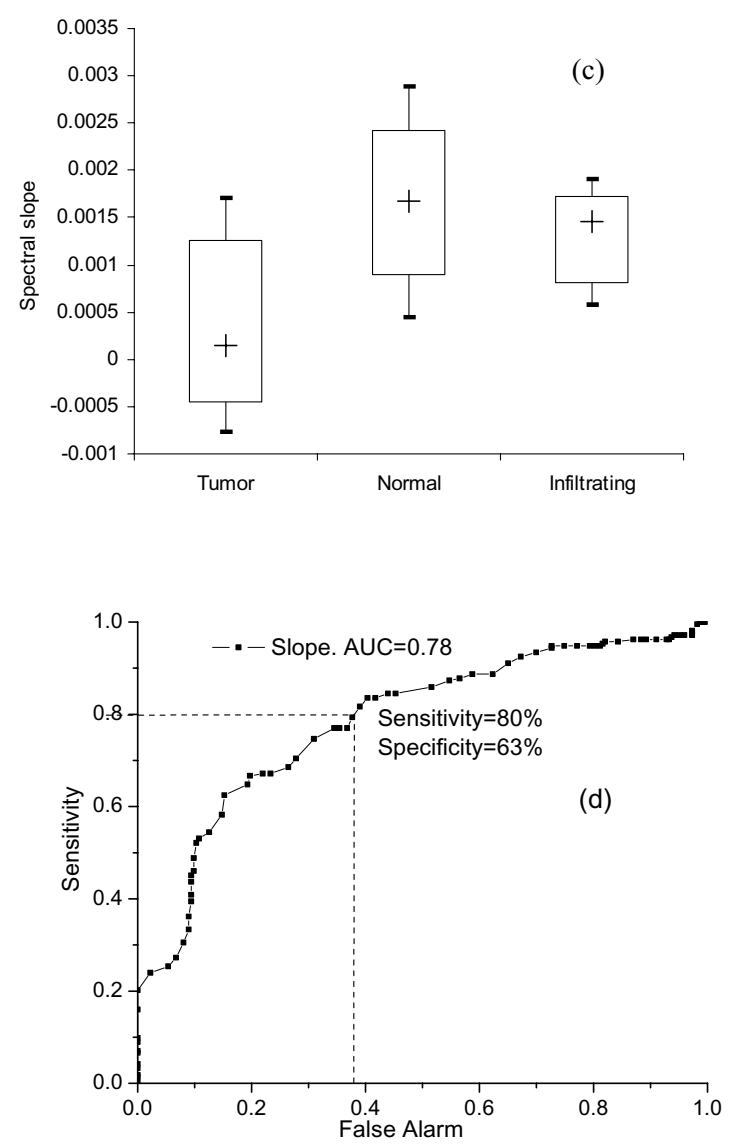

Fig. 2c, d. ELS spectra and statistical analysis of spectral slopes for three types of brain tissue. (a) Typical ELS spectra for normal brain tissue, tumor, and tumor-infiltrated brain tissue. The three types of tissue samples were obtained from a single patient. (b) Statistics of ELS spectral slopes for three tissue types from a single patient. Box: 1 and 3 quartiles; bars: mean \pm standard deviation; cross: median. (c) Statistics of ELS spectral slopes of the spectra from all the patients as listed in Table 1. (d) ROC curve of using spectral slope as a diagnostic marker

ELS spectra from a single normal or tumor core sample were used as testing data set, and ELS spectra from all other tumor or normal samples were used for ANN training. This test was then repeated to each normal or tumor sample. By changing the cut-off threshold for simulated scores, the ROC curve was calculated and is shown in Fig. 4(b). Its AUC value is 0.92 , and a typical pair of sensitivity and specificity is $80 \%$ and 93\%. In order to evaluate how the ANN classifier characterizes infiltrating tissue, we tested the ELS spectra measured from infiltrating samples against ANN trained with normal and tumor samples. Interestingly, as shown in Fig. 4(c) and (d), the output scores of tumorinfiltrated brain samples may classify them as tumor, normal, or in-between (with an output score close to 

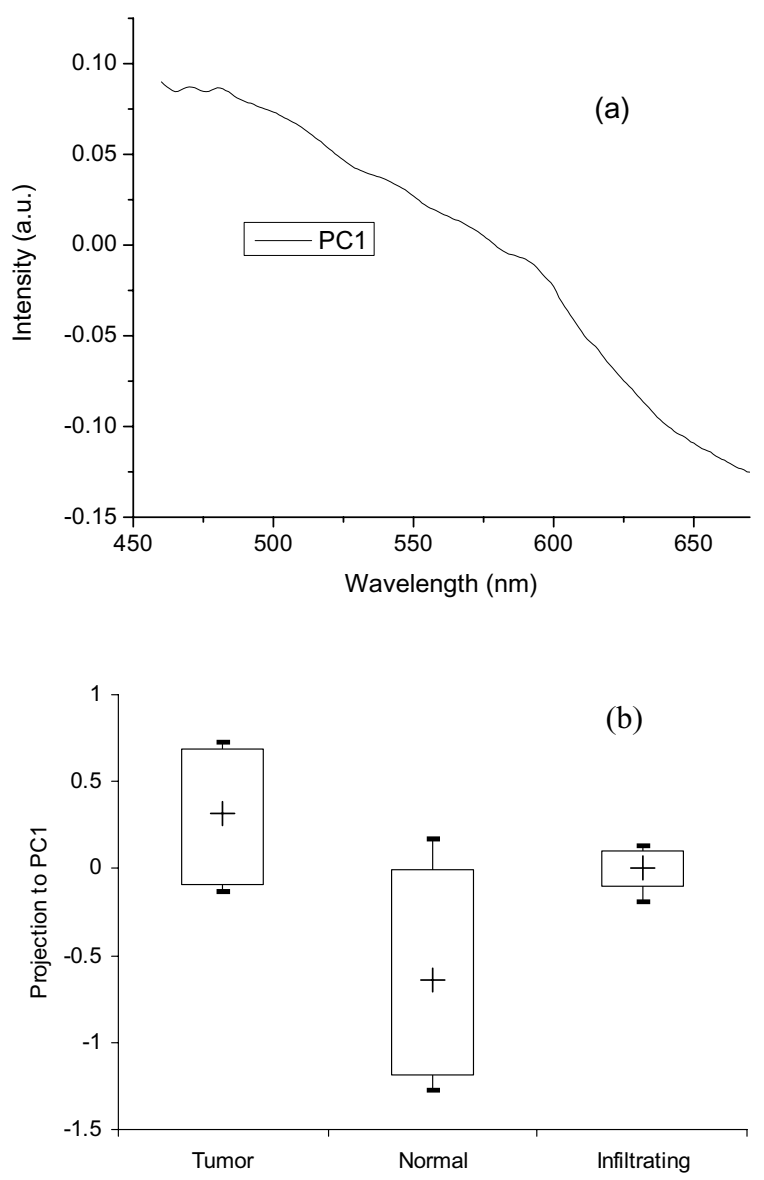

Fig. 3a, b. PCA analysis of the ELS spectra from three types of brain tissue. (a) Spectrum of PC1 component. b) Statistics of PC1 projection coefficients for three tissue types from a single patient. (c) Statistics of PC1 projection coefficients for three tissue types from all the patients listed in Table 1. (d) ROC curve of using PC1 projection coefficients as a diagnostic marker

\section{$0.5)$ tissue types.}

Table 2 compares the performance metrics, including AUC values from ROC curves, sensitivity, and specificity, of the three classification markers mentioned above. We note that while slope and PC1 projection give fair diagnostic accuracy, ANN output scores work as an excellent marker.

\section{Conclusion and discussion}

In this paper, we report a pilot study designed to test elastic light-scattering (ELS) spectroscopy for characterizing normal, tumor, and tumor-infiltrated brain tissues. ELS spectra were measured on 390 histology sites on 36 ex vivo tissue specimen from 29 patients undergoing elective cranial surgery. We employed and
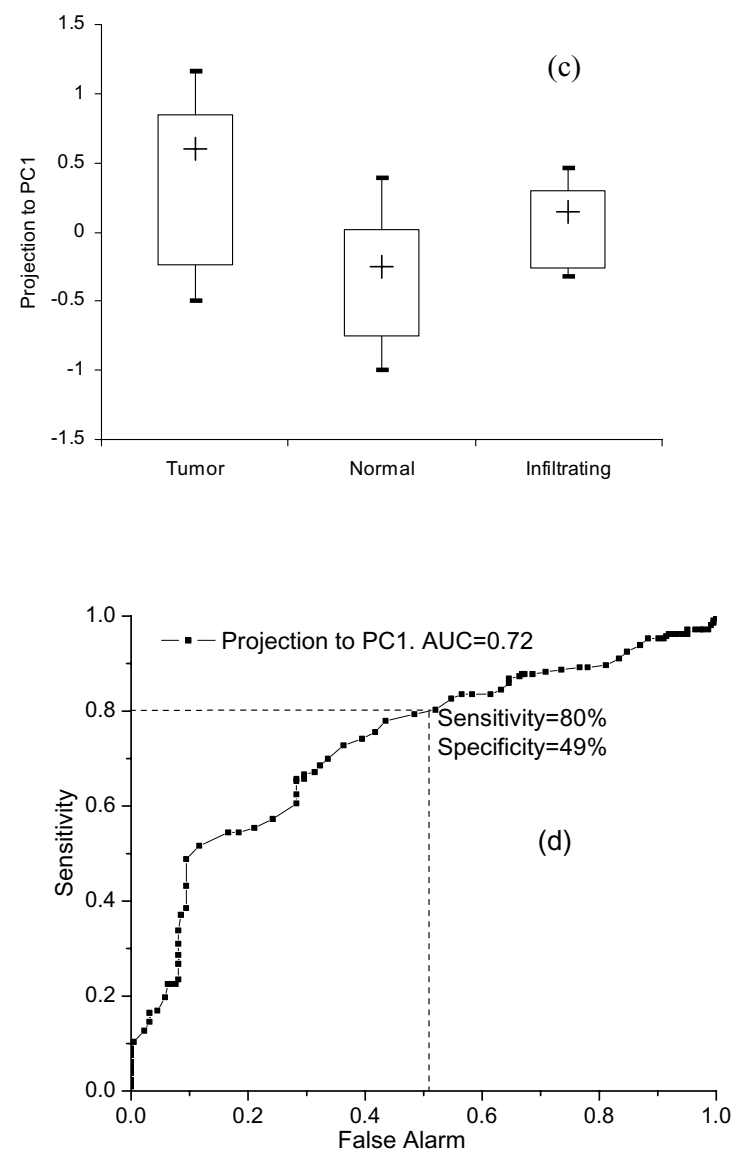

Fig. 3c, d. PCA analysis of the ELS spectra from three types of brain tissue. (a) Spectrum of PC1 component. b) Statistics of PC1 projection coefficients for three tissue types from a single patient. (c) Statistics of PC1 projection coefficients for three tissue types from all the patients listed in Table 1. (d) ROC curve of using PC1 projection coefficients as a diagnostic marker

compared the performance of three methods of spectral classification for tissue characterization, including spectral slope analysis, principle component analysis (PCA), and artificial neural network (ANN) classification. The ANN classifier yields the best correlation between spectral pattern and histopathological diagnosis, with a typical sensitivity of $80 \%$ and specificity of $93 \%$ for differentiating tumor from normal brain tissue. Interestingly, we also demonstrate that all three classification methods have the potential to identify and quantitatively characterize tumor-infiltrated brain tissues.

\section{Acknowledgements}

The authors would like to thank Dr. Vadim Backman and his group members at Northwestern University for 

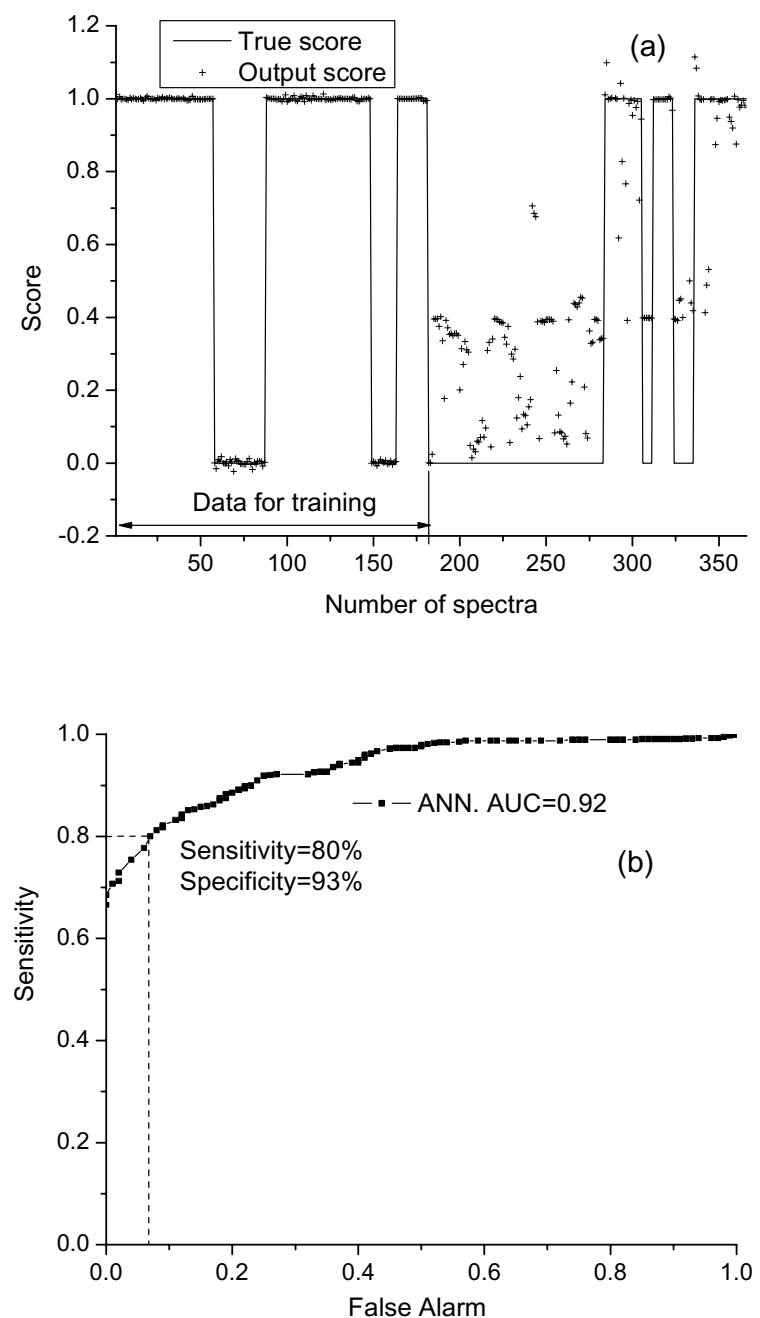

Fig. 4a, b. ANN analysis results. (a) ANN output scores for a leave-half test. Here, measurement \#1 through \#183 were used as training data set, while measurement \#184 through \#366 were used as test data set. b) ROC curve calculated using leave-one-sample test of ANN analysis for classification. Here, ANN classification was repeated for each sample while using ELS spectra of all other samples as training data. (c) ANN output scores for using all normal and tumor measurements as training set and all tumor-infiltrated brain tissue samples as test set. (d) Same as (c) with alternative $\mathrm{x}$-axis range emphasizing the tumor-infiltrated brain tissue type

providing the ELS system for our measurements and Drs. Joshua M. Rosenow and James P. Chandler for contributing tissue specimen.

\section{References}

[1] R. Stupp, W.P. Mason, M.J. van den Bent, M. Weller, B. Fisher, M.J.B. Taphoorn, K. Belanger, A.A. Brandes, C. Marosi and U. Bogdahn, Radiotherapy plus Concomitant and Adjuvant
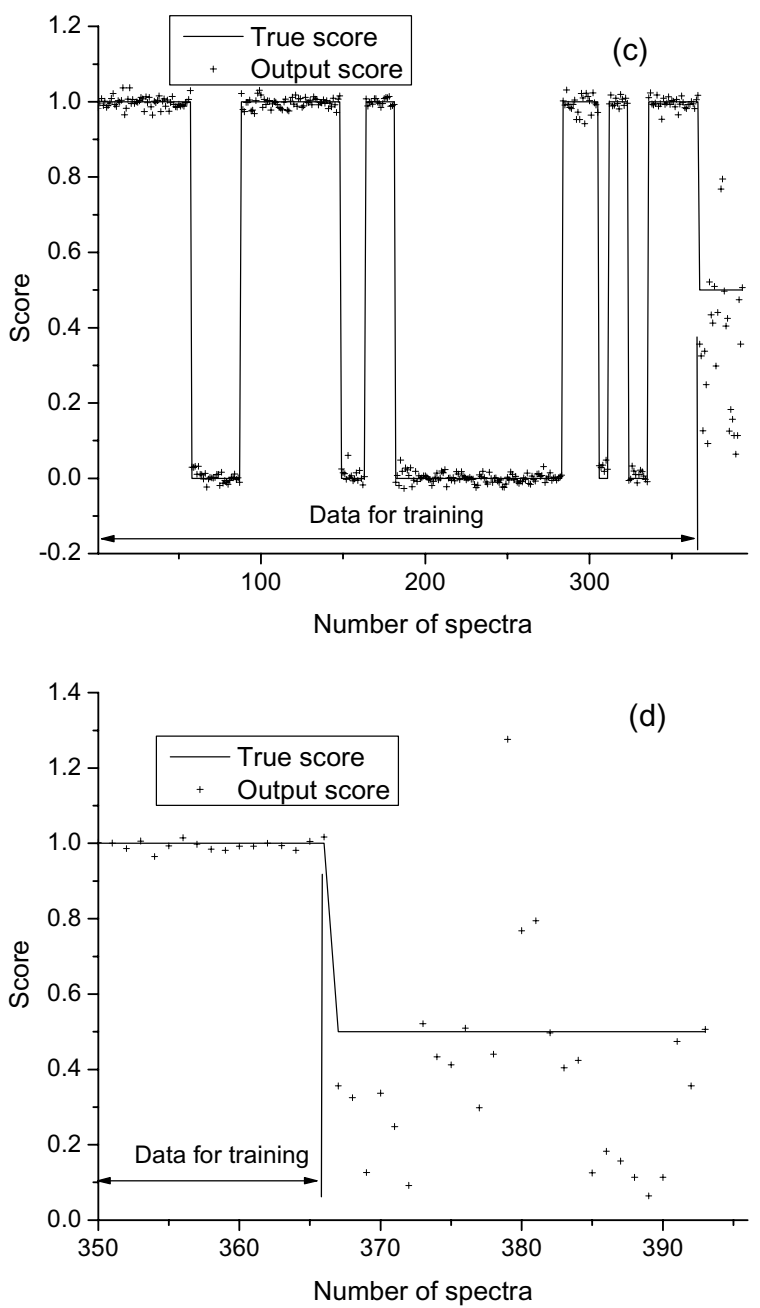

Fig. 4c, d. ANN analysis results. (a) ANN output scores for a leave-half test. Here, measurement \#1 through \#183 were used as training data set, while measurement \#184 through \#366 were used as test data set. b) ROC curve calculated using leave-one-sample test of ANN analysis for classification. Here, ANN classification was repeated for each sample while using ELS spectra of all other samples as training data. (c) ANN output scores for using all normal and tumor measurements as training set and all tumor-infiltrated brain tissue samples as test set. (d) Same as (c) with alternative $\mathrm{x}$-axis range emphasizing the tumor-infiltrated brain tissue type

Temozolomide for Glioblastoma, New England Journal of Medicine 352 (2005), 987.

[2] J. Wisoff, J. Boyett, M. Berger, C. Brant, H. Li, A. Yates, P. McGuire-Cullen, P. Turski, L. Sutton, J. Allen, R. Packer and J. Finlay, Current Neurosurgical Management and the Impact of the Extent of Resection in the Treatment of Malignant Gliomas of CHildhood: A Report of the Children's Cancer Group Trial no. CCG-945, J Neurosurg 90 (1998), 1147-1148.

[3] M. Lacroix, D. Abi-Said, D. Fourney, Z. Gokaslan, W. Shi, F. DeMonte, F. Lang, I. McCutcheon, S. Hassenbusch, E. Holland, K. Hess, C. Michael, D. Miller and R. Sawaya, A Multivariate Analysis of 416 Patients with Glioblastoma Multi- 
forme: Prognosis, Extent of Resection, and Survival, J Neurosurg 95 (2001), 190-198.

[4] J. Regelsberger, F. Lohmann, K. Helmke and M. Westphal, Ultrasound-Guided Surgery of Deep Seated Brain Lesions, Eruopean Journal of Ultrasound 12 (2000), 115-121.

[5] P. LeRoux, T. Winter, M. Berger, L. Mack, K. Wang and J. Elliott, A Comparison Between Preoperative Magnetic Resonance and Intraoperative Ultrasound Tumor Volumes and Margins, J Clin Ultrasound 22 (1994), 29-36.

[6] P. LeRoux, M. Berger, G. Ojemann, K. Wang and L. Mack, Correlation of Intraoperative Ultrasound Tumor Volumes and Margins with Preoperative Computerized Tomography Scans. An Intraoperative Method to Enhance Tumor Resection, $J$ Neurosurg 71 (1989), 691-698.

[7] R. Comeau, A. Sadikot, A. Fenster and T. Peters, Intraoperative Ultrasound for Guidance and Tissue Shift Correction in Image-Guided Neurosurgery, Med Phys 27 (2000), 787-800.

[8] M. Apuzzo and J. Sabashin, Computed tomographic guidance stereotaxis in the management of intracranial mass lesions, Neurosurgery 12 (1983), 277-285.

[9] L. Lunsford, R. Parrish and L. Albright, Intraoperative imaging with a therapeutic computed tomographic scanner, Neurosurgery 15 (1984), 559-561.

[10] H. Okudera, T. Takemae and S. Kobayashi, Intraoperative Computed Tomographic Scanning During Transsphenoidal Surgery: Technical Note, Neurosurgery 32 (1993), 10411043.

[11] M. Shalit, Y. Israeli, S. Matz and M. Cohen, Intraoperative computerized axial tomography, Surg Neurol 11 (1979), 382384.

[12] C. Wirtz, F. Albert, M. Schwaderer, C. Heuer, A. Staubert, V. Tronnier, M. Knauth and S. Kunze, The Benefit of Neuronavigation for Neurosurgery Analyzed by its Impact on Glioblastoma Surgery, Neurol Res 22 (2000), 354-360.

[13] A. Nabavi, P. M. Black, D. T. Gering, C.-F. Westin, V. Mehta, R.S.J. Pergolizzi, M. Ferrant, S. K. Warfield, N. Hata, R.B. Schewartz, W.M.I. MWells, R. Kikinis and F.A. Jolesz, Serial Intraoperative Magnetic Resonance Imaging of Brain Shift, Neurosurgery 48 (2001), 787-798.

[14] P.M. Black, E.I. Alexander, C. Martin, T. Moriarty, A. Nabavi, T.A. Wong, R.B. Schwartz and F.A. Jolesz, Craniotomy for Tumor Treatment in an Intraoperative Magnetic Resonance Imaging Unit, Neurosurgery 45 (1999), 423.

[15] M. Schulder, D. Liang and P. Carmel, Cranial Surgery Navigation Aided by a Compact Intraoperative Magnetic Resonance Imager, J Neurosurg 94 (20010, 936-945.

[16] M. Hadani, R. Spiegelman, Z. Feldman, H. Berkenstadt and Z. Ram, Novel, Compact, Intraoperative Magnetic Resonance Imaging-Guided System for Conventional Neurosurgical Operating Rooms, Neurosurgery 48 (2001), 799-809.

[17] R.J. Bohinski, A.K. Kokkino, R.E. Warnick, M.F. GaskillShipley, D.W. Kormos, R.R. Lukin and J.M.J. Tew, Glioma Resection in a Shared-Resource Magnetic Resonance Operating Room After Optimal Image-Guided Frameless Stereotactic Resection, Neurosurgery 48 (2001), 731-744.

[18] F.I.V. Earnest, P.J. Kelly, B. Scheithauer, B.A. Kall, T.L. Cascino, R.L. Ehman, G.S. Forbes and P.L. Axley, Cerebral astrocytomas: Histopathologic correlation of MR and CT contrast enhancement with stereotactic biopsy, Radiology 166 (1988), 823-827.

[19] O. Ganslandt, A. Stadlbauer, R. Fahlbusch, K. Kamada, R. Buslei, I. Blumcke, E. Moser and C. Nimsky, Proton magnetic resonance spectroscopic imaging integrated into image-guided surgery: Correlation to standard magnetic resonance imaging and tumor cell density, Neurosurgery 56 (2005), 291-298.

[20] I. Park, G. Tamai, M.C. Lee, C.F. Chuang, S.M. Chang, M.S. Berger, S.J. Nelson and A. Pirzkall, Patterns of Recurrence Analysis in Newly Diagnosed Glioblastoma Multiforme After Three-Dimensional Conformal Radiation Therapy With Respect to Pre-Radiation Therapy Magnetic Resonance Spectroscopic Findings, International Journal of Radiation Oncology, Biology, Physics 69 (2007), 381-389.

[21] I.J. Bigio, S.G. Bown, G. Briggs, C. Kelley, S. Lakhani, D. Pickard, P.M. Ripley, I.G. Rose and C. Saunders, Diagnosis of breast cancer using elastic-scattering spectroscopy: preliminary clinical results, Journal of Biomedical Optics 5 (2000), 221.

[22] T.M. Breslin, F. Xu, G.M. Palmer, C. Zhu, K.W. Gilchrist and N. Ramanujam, Autofluorescence and Diffuse Reflectance Properties of Malignant and Benign Breast Tissues, Soc Surgical Oncol 11 (2004), 65-70.

[23] K.S. Johnson, D.W. Chicken, D.C.O. Pickard, A.C. Lee, G. Briggs, M. Falzon, I.J. Bigio, M.R. Keshtgar and S.G. Bown, Elastic scattering spectroscopy for intraoperative determination of sentinel lymph node status in the breast, Journal of Biomedical Optics 9 (2004), 1122.

[24] B.J. Tromberg, N. Shah, R. Lanning, A. Cerussi, J. Espinoza, T. Pham, L. Svaasand and J. Butler, Non-Invasive In Vivo Characterization of Breast Tumors Using Photon Migration Spectroscopy, Neoplasia 2 (2000), 26-40.

[25] C. Zhu, G. M. Palmer, T. M. Breslin, F. Xu and N. Ramanujam, Use of a multiseparation fiber optic probe for the optical diagnosis of breast cancer, Journal of Biomedical Optics $\mathbf{1 0}$ (2005), 024032.

[26] S.K. Chang, Y.N. Mirabal, E.N. Atkinson, D. Cox, A. Malpica, M. Follen and R. Richards-Kortum, Combined reflectance and fluorescence spectroscopy for in vivo detection of cervical pre-cancer, Journal of Biomedical Optics 10 (2005), 024031.

[27] I. Georgakoudi, E.E. Sheets, M.G. Muller, V. Backman, C.P. Crum, K. Badizadegan, R.R. Dasari and M.S. Feld, Trimodal spectroscopy for the detection and characterization of cervical precancers in vivo, Am J Obstet Gynecol 186 (2002), 374-382.

[28] N. Ramanujam, M.F. Mitchell, A. Mahadevan, S. Warren, S. Thomsen, E. Silva and R. Richards-Kortum, In vivo Diagnosis of Cervical Intraepithelial Neoplasia Using 337-nmExcited Laser-Induced Fluorescence, Proceedings of the $\mathrm{Na}$ tional Academy of Sciences of the United States of America 91 (1994), 10193-10197.

[29] H.K. Roy, Y.L. Kim, Y. Liu, R.K. Wali, M.J. Goldberg, V. Turzhitsky, J. Horwitz and V. Backman, Risk stratification of colon carcinogenesis through enhanced backscattering spectroscopy analysis of the uninvolved colonic mucosa, Clin Cancer Res 19 (2006), 961-968.

[30] H.K. Roy, Y. Liu, R.K. Wali, Y.L. Kim, A.K. Kromine, M.J. Goldberg and V. Backman, Four-dimensional elastic lightscattering fingerprints as preneoplastic markers in the rat model of colon carcinogenesis, Gastroenterology 126 (2004), 1071-1081.

[31] Y. Liu, R.E. Brand, V. Turzhitsky, Y.L. Kim, H.K. Roy, N. Hasabou, C. Sturgis, D. Shah, C. Hall and V. Backman, Optical Markers in Duodenal Mucosa Predict the Presence of Pancreatic Cancer, Clinical Cancer Research 13 (2007), 4392.

[32] S.A. Boppart, M.E. Brezinski, C. Pitris and J.G. Fujimoto, Optical coherence tomography for neurosurgical imaging of human intracortical melanoma, Neurosurgery 43 (1998), 834841. 
[33] S.A. Boppart, Optical coherence tomography: Technology and applications for neuroimaging, Psychophysiology $\mathbf{4 0}$ (2003), 529-541.

[34] H.J. Böhringer, D. Boller, J. Leppert, U. Knopp, E. Lankenau, E. Reusche, G. Hüttmann and A. Giese, Time-domain and spectral-domain optical coherence tomography in the analysis of brain tumor tissue, Lasers in Surgery and Medicine $\mathbf{3 8}$ (2006), 588-597.

[35] W. Stummer, A. Novotny, H. Stepp, C. Goetz, K. Bise and H.J. Reulen, Fluorescence-guided resection of glioblastoma multiforme by using 5-aminolevulinic acid-induced porphyrins: a prospective study in 52 consecutive patients, J Neurosurg 93 (2000), 1003-1013,

[36] J. Shinoda, H. Yano, S. Yoshimura, A. Okumura, I. Kaku and N.S. Sakai, Fluorescence-guided resection of glioblastoma multiforme by using high-dose fluorescein sodium, $J$. Neurosurg 99 (2003), 597-603.

[37] W. Stummer, U. Pichlmeier, T. Meinel, O. D. Wiestler, F. Zanella and H.J. Reulen, Fluorescence-guided surgery with 5-aminolevulinic acid for resection of malignant glioma: a randomised controlled multicentre phase III trial, Lancet Oncology 7 (2006), 392-401.

[38] M.F. Kircher, U. Mahmood, R.S. King, R. Weissleder and L. Josephson, A Multimodal Nanoparticle for Preoperative Magnetic Resonance Imaging and Intraoperative Optical Brain Tumor Delineation, AACR 63 (2003), 8122-8125.

[39] R. Trehin, J.L. Figueiredo, M.J. Pittet, R. Weissleder, L. Josephson and U. Mahmood, Fluorescent nanoparticle uptake for brain tumor visualization, Neoplasia 8 (2006), 302-311.

[40] G. Bottiroli, A.C. Croce, D. Locatelli, R. Nano, E. Giombelli, A. Messina and E. Benericetti, Brain tissue autofluorescence: an aid for intraoperative delineation of tumor resection margins, Cancer Detect Prev 22 (1998), 330-339.

[41] A.C. Croce, S. Fiorani, D. Locatelli, R. Nano, M. Ceroni, F. Tancioni, E. Giombelli, E. Benericetti and G. Bottiroli, Diagnostic Potential of Autofluorescence for an Assisted Intraoperative Delineation of Glioblastoma Resection Margins, Photochemistry and Photobiology 77 (2002), 309-318.

[42] W.H. Yong, P.V. Butte, B.K. Pikul, J.A. Jo, Q. Fang, T. Papaioannou, K. Black and L. Marcu, Distinction of brain tissue, low grade and high grade glioma with time-resolved fluorescence spectroscopy, Front. Biosci 11 (2006), 1255-1263.

[43] W.C. Lin, S.A. Toms, M. Motamedi, E.D. Jansen and A. Mahadevan-Jansen, Brain tumor demarcation using optical spectroscopy; an in vitro study, Journal of Biomedical Optics 5 (2000), 214.

[44] W.C. Lin, S.A. Toms, M. Johnson, E.D. Jansen and A. Mahadevan-Jansen, In Vivo Brain Tumor Demarcation Using Optical Spectroscopy, Photochemistry and Photobiology $\mathbf{7 3}$ (2001), 396-402.

[45] S.C. Gebhart, W.C. Lin and A. Mahadevan-Jansen, In vitro determination of normal and neoplastic human brain tissue optical properties using inverse adding-doubling, Phys Med Biol 51 (2006), 2011-2027.

[46] S.A. Toms, W.C. Lin, R.J. Weil, M.D. Johnson, E.D. Jansen and A. Mahadevan-Jansen, Intraoperative optical spectroscopy identifies infiltrating glioma margins with high sensitivity, Neurosurgery 57 (2005), 382-391.

[47] X. Li, A. Taflove and V. Backman, Recent progress in exact and reduced-order modeling of light-scattering properties of complex structures, Selected Topics in Quantum Electronics, IEEE Journal of 11 (2005), 759-765.

[48] X. Li, A. Taflove and V. Backman, Anomalous oscillations in the spectra of light backscattered by inhomogeneous microparticles, Physical Review E 75 (2007), 37601.

[49] Y.L. Kim, Y. Liu, R.K. Wali, H.K. Roy, M.J. Goldberg, A.K. Kromin, K. Chen and V. Backman, Simultaneous measurement of angular and spectral properties of light scattering for characterization of tissue microarchitecture and its alteration in early precancer, Selected Topics in Quantum Electronics, IEEE Journal of 9 (2003), 243-256.

[50] Y.L. Kim, Y. Liu, R.K. Wali, H.K. Roy and V. Backman, Lowcoherent backscattering spectroscopy for tissue characterization, Applied Optics 44 (2005), 366-377.

[51] V. Backman, R. Gurjar, K. Badizadegan, I. Itzkan, R.R. Dasari, L.T. Perelman and M.S. Feld, Polarized light scattering spectroscopy for quantitativemeasurement of epithelial cellular structures in situ, Selected Topics in Quantum Electronics, IEEE Journal of 5 (1999), 1019-1026.

[52] R.S. Gurjar, V. Backman, L.T. Perelman, I. Georgakoudi, K. Badizadegan, I. Itzkan, R.R. Dasari and M.S. Feld, Imaging human epithelial properties with polarized light-scattering spectroscopy, Nat Med 7 (2001), 1245-1248.

[53] M.P. Siegel, Y.L. Kim, H.K. Roy, R.K. Wali and V. Backman, Assessment of blood supply in superficial tissue by polarization-gated elastic light-scattering spectroscopy, Applied Optics 45 (2006), 335-342. 


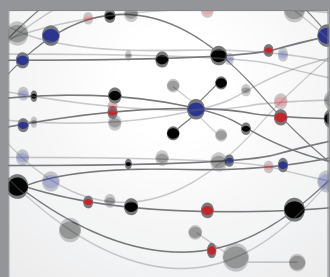

The Scientific World Journal
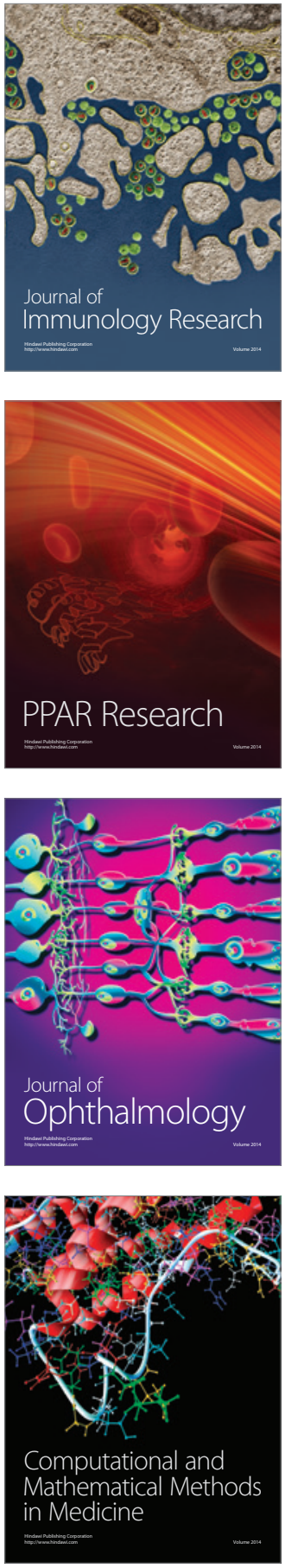

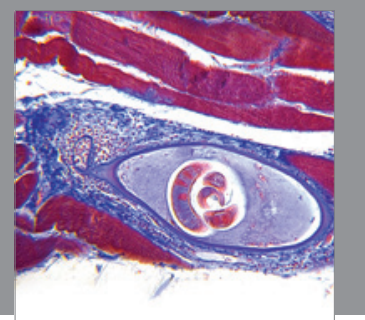

Gastroenterology

Research and Practice
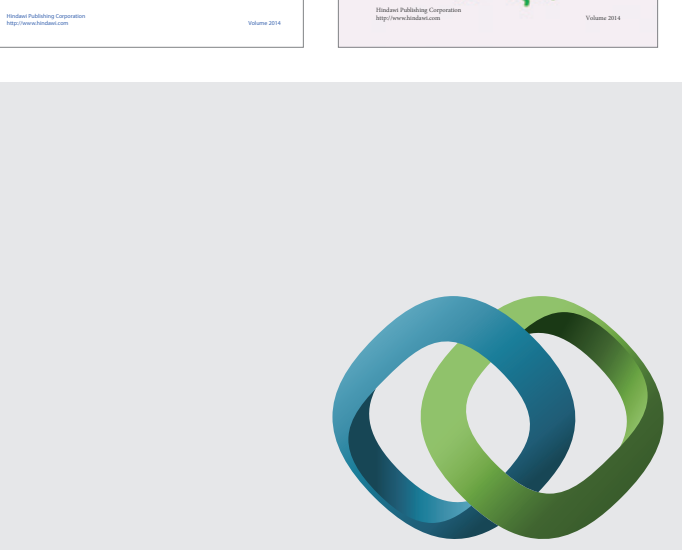

\section{Hindawi}

Submit your manuscripts at

http://www.hindawi.com
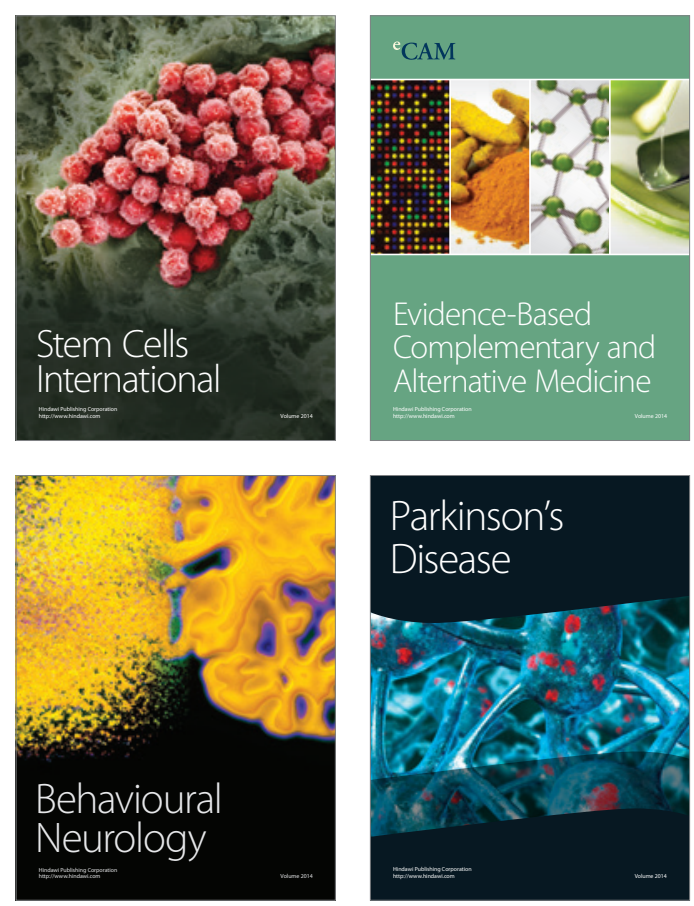

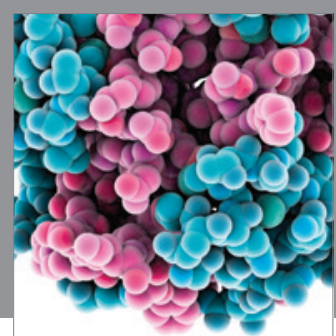

Journal of
Diabetes Research

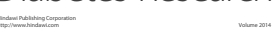

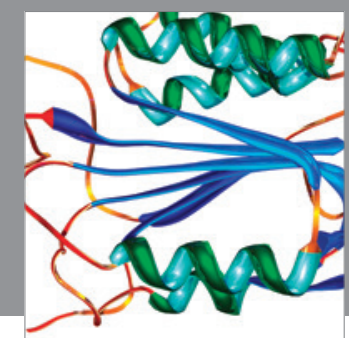

Disease Markers
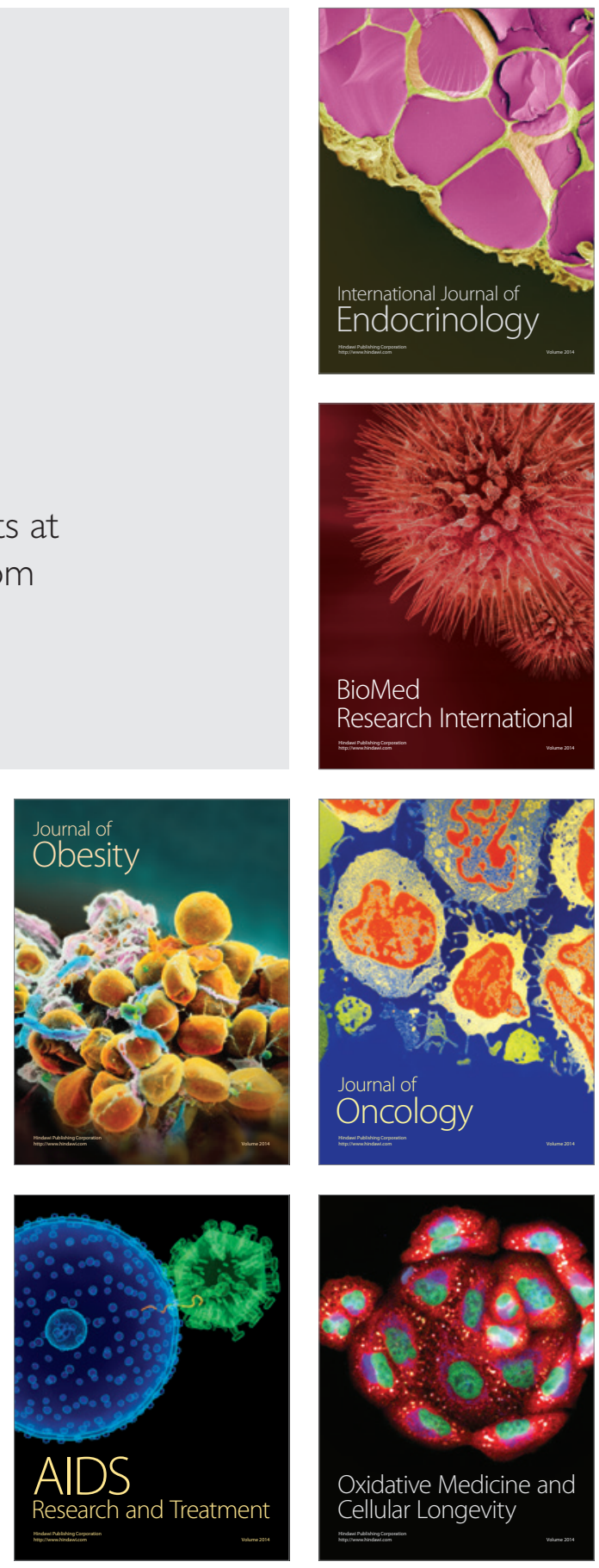